\title{
Komplikationen der intertrochantären Frakturen
}

\author{
Jan Bartoníček
}

Die instabilen intertrochantären Frakturen (A0 31 A3) werden durch die Form des proximalen Fragments und den Verlauf der Bruchlinie charakterisiert. Die Hauptbruchlinie startet an der lateralen Seite distal vom Tuberculum innominatum und verläuft proximolateral, das heißt umgekehrt wie bei pertrochantären Frakturen. Deshalb wird dieser Bruchtyp auch als „reversed fracture“ bezeichnet.

Diese Brüche werden immer öfter mit einem intramedullären Kraftträger behandelt [3]. Ein häufiges Problem bei der Operation stellt die Reposition dar, manchmal ist eine offene Reposition erforderlich. Die häufigsten postoperativen Komplikationen sind die mechanischen Probleme, die auch zum Implantatversagen wie Bruch des Nagels führen [1] können. Wenig bekannt ist aber eine weitere, sehr ernsthafte Komplikation, die Gegenstand dieser Mitteilung ist.

\section{Kasuistik}

Ein Mann, 60 Jahre alt, chronischer Raucher und Alkoholiker, erlitt im Juli 2006 einen intertrochantären Bruch. Einen Tag nach dem Unfall erlitt er einen epileptischen Anfall mit Herzstillstand. Er wurde deshalb eine Woche nach dem Unfall operiert. Die Operation dauerte 50 Minuten inklusive der Reposition auf dem Extensionstisch. Die Fraktur wurde mit einem intramedullären Nagel (PFHMedin) fixiert. Die Lage der Kopfschrauben im Femurkopf war optimal in beiden Projektionen (a.-p., axial). Der Nagel wurde distal dynamisch verriegelt.

Auf der postoperativen Aufnahme war die Zertrümmerung des Trochanter major gut erkennbar. Der Patient wurde am 2. Tag nach der Operation mobilisiert.

OP-JOURNAL 2008; 24: 214-215

(C) Georg Thieme Verlag KG Stuttgart • New York DOI 10.1055/s-2008-1039075
Die Operationswunde heilte ohne Komplikationen, der Patient wurde nach 14 Tagen entlassen. Bei der Kontrolle nach 2 Monaten (September 2006) wurde ein nur minimaler Durchbau des Bruches festgestellt, eine partielle Resorption des Schenkelhalses und Protrusion einer der Kopfschrauben in den Gelenkspalt. Der Patient wurde sofort zur Reoperation aufgenommen, allerdings kam es zum deliranten Anfall, deshalb wurde der Eingriff verschoben. In Anbetracht des Gesamtzustands wurde 5 Monate (November 2006) nach der Operation nur die perforierte Schraube entfernt. Der Patient wurde wiederholt kontrolliert und eine schrittweise Frakturheilung war erkennbar. Trotz wiederholter Hinweise hatte der Patient das Bein ohne Krücken voll belastet. Neun Monate (April 2007) nach der Operation kam es zum Versagen der Osteosynthese. Nach gründlicher Vorbereitung wurde eine zementlose Totalendoprothese (Spotorno-Pfanne, Wagner-Revisionsschaft) implantiert. Der Patient wurde 12 Tage nach der Operation nach Hause entlassen. Eine Woche nach der Entlassung (Mai 2007) wurde er mit einer Luxation der Endoprothese wieder aufgenommen. Eine geschlossene Reposition wurde durchgeführt. Fünf Tage nach dieser verließ der Patient auf eigenen Wunsch das Krankenhaus. Tags darauf wurde er mit einer Reluxation und Wunddehiszenz erneut eingewiesen. Es wurden eine Reposition und Wundrevision durchgeführt. Im Heilverlauf kam es zu 2 weiteren Luxationen. Nach Wundheilung wurde der Patient nach Hause entlassen.

Einen Monat danach (Juni 2007) wurde er im alkoholisierten Zustand mit einer periprothetischen Fraktur der Femurdiaphyse erneut aufgenommen. Die Fraktur wurde mit einer Winkelplatte stabilisiert. Nach 14 Tagen kam es zu einer wiederholten Wunddehiszenz (Juli 2007). Seit dem Primärunfall war zu diesem Zeitpunkt genau ein Jahr ver- gangen. Die Wunde heilte nach der Revision. Eine bislang letzte Kontrolle erfolgte im Oktober 2007, wobei eine Heilung der periprothetischen Fraktur sichtbar war. $\mathrm{Zu}$ weiteren Kontrollen hat sich der Patient nicht mehr eingefunden (Abb.1 a bis $\mathbf{n}$ ).

\section{Diskussion}

Zur Entstehung der Komplikationen haben im vorliegenden Fall sowohl der Allgemeinzustand als auch die fehlende Compliance des Patienten wesentlich beigetragen. Als Hauptursache für das Osteosyntheseversagen betrachten wir allerdings, aufgrund der Erfahrungen mit 4 ähnlichen Fällen, den Bruchtyp. Diese 4 Patienten wurden ebenfalls mit einem Marknagel behandelt. In allen Fällen war der Trochanter major vertikal gespalten. Zugleich wurde durch eine weitere Bruchlinie die Basis des Femurhalses von Trochanter major abgetrennt. Bei allen Patienten kam es zur Resorption der Halsbasis und anschließendem Kollaps der Fragmente ohne Frakturheilung. In 3 Fällen wurde die Situation mit einer Totalendoprothese gelöst. In einem Fall verhinderte dies der Gesamtzustand des Patienten.

In der Literatur fanden wir nur eine Arbeit, die sich mit diesem Problem befasst. Kyle et al. [6] haben aufgrund der Erfahrungen mit einer Gruppe von 20 Patienten auf diesen besonderen Typ der trochantären Fraktur hingewiesen, welcher mit einer Fraktur des Schenkelhalses verbunden ist. Alle Brüche wurden mit DHS behandelt. Bei 5 Patienten $(25 \%)$ kam es zum Kollaps und zum Osteosyntheseversagen. Die Autoren empfahlen deshalb die Implantation einer Endoprothese statt der Osteosynthese dieser Brüche mit DHS. Weder aus dem Text noch aus den Röntgenbildern ist der Typ der trochantären Frakturen in der Serie von Kyle klar ersichtlich, am ehesten handelt es sich um den Bruchtyp 31 A3. 


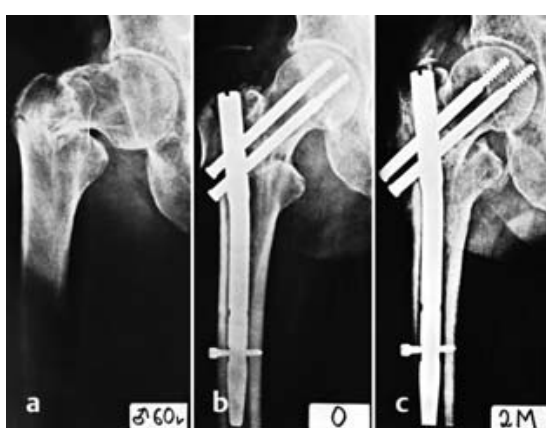

Abb. 1a bis c a Intertrochantäre Fraktur mit Fraktur des Schenkelhalses. b Osteosynthese mit PFH-Nagel. c Zwei Monate später Perforation der distalen Kopfschraube in die Gelenklinie.

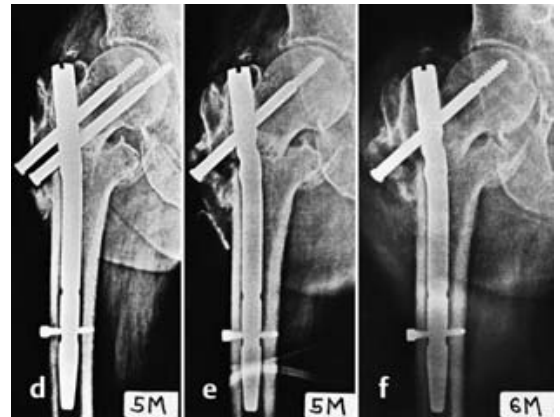

Abb. 1 d bis f d Nach 5 Monaten Progredienz der Perforation. e Situation nach Schraubenentfernung. f Nach 6 Monaten blieb die Position der Fragmente unverändert.

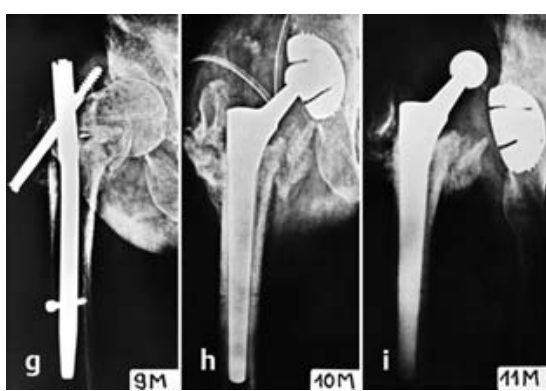

Abb. $\mathbf{1 g}$ bis i g Mechanisches Versagen nach 9 Monaten. $\mathbf{h}$ Implantation einer Totalendoprothese mit Spotorno-Pfanne und Wagner-Revisionschaft. i Ein Monat später Luxation der Prothese.

\section{Literatur}

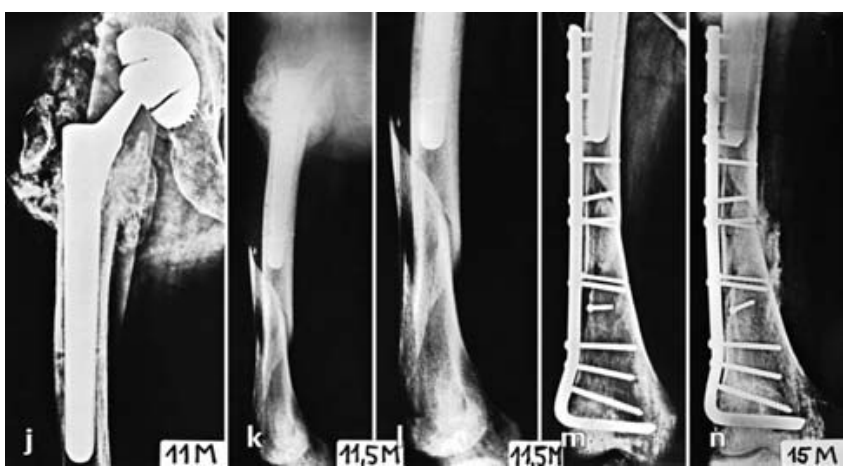

Abb. $1 \mathbf{j}$ bis $\mathbf{n}$ j Zustand nach Reposition. $\mathbf{k}$, I Einen Monat danach periprothetische Femurschaftfraktur. m Osteosynthese mit kondylärer Platte. n Frakturheilung 3,5 Monate später.

Dieser Typ des intertrochantären Bruches, bei dem die sekundäre Fakturlinie in den großen Trochanter hineinzieht, wird in der AO-Klassifikation als $31 \mathrm{~A} 3$ bezeichnet. In Seinsheimers Klassifikation [7] subtrochantärer Brüche entspricht er dem Typ V, in Waddells Klassifikation der subtrochantären Frakturen [8] dem Typ III. Alle 5 unserer Fälle gehörten in diese Gruppe.

Kyle et al. [6] erklärten die Kollapsursachen nicht. Unserer Meinung nach kam es zur Schädigung der Blutversorgung des basalen Anteils des Schenkelhalses und nachfolgender Resorption [5]. Für die Theorie der Durchblutungsstörung sprechen auch die Probleme bei der Frakturheilung. Die trochantären Frakturen heilen normalerweise im Verlauf von 3 Monaten. Eine gleichzeitige Femurkopfnekrose, die bei intertrochantären Brüchen sehr selten ist, haben wir jedoch nur in einem Fall verzeichnet. Zur Schädigung der Blutversorgung im Bereich der Halsbasis und des großen Trochanters könnte auch der intermedulläre Nagel beitragen [4].
Aufgrund dieser Erfahrung haben wir bei 2 weiteren Patienten ( 23 und 45 Jahre) eine DHS mit Trochanterabstützplatte bei diesem Bruchtyp benutzt. Bei der Operation sind wir maximal schonend vorgegangen. Obwohl es sich um junge Patienten handelte, ging die Frakturheilung nur sehr langsam voran, die radiologischen Bruchlinien verschwanden erst nach einem Jahr. Allerdings kam es weder zur einer Resorption des Schenkelhalses noch zur Kopfnekrose.

\section{Fazit}

Die intertrochantären Frakturen mit Zertrümmerung des Trochanter major und gleichzeitigem basozervikalen Schenkelhalsbruch kann man als risikoreichen Bruchtyp bezeichnen. Der Grund ist vermutlich die Schädigung der Blutversorgung des proximalen Femurs im Bereich des Trochanter major und der Schenkelhalsbasis. Deshalb sollten diese Brüche nicht mit einem Marknagel behandelt werden, sondern mit DHS und Trochanterabstützplatte, da die alleinige Osteosynthese mit DHS keine ausreichende Stabilität bietet.
1 Bartoníček J, Douša P, Krbec M. [Complications of the fixation of proximal femur fractures by gamma nail]. Acta Chir Orthop Traumatol Cech 1998; 65: 84-99

2 Bartoníček J, Frič V, Skála-Rosenbaum J, Douša P. Avascular necrosis of the femoral head in pertrochanteric fractures: a report of 8 cases and a review of the literature. J Orthop Trauma 2007; 21: 229-236

3 Dávid A, von der Heyde D, Pommer A. Therapiemöglichkeit trochantärer Frakturen. Orthopäde 2000; 29: 294-301

${ }^{4}$ Gautier E, Ganz K, Krugel N, Gill T, Ganz R. Anatomy of the medial femoral circumflex artery and its surgical implication. J Bone Joint Surg [Br] 1999; 82: 679-683

5 Judet J, Judet R, Lagrange J, Dunoyer J. A study of the arterial vascularization of the femora neck in the adult. J Bone Joint Surg [Am] 1955; 37: 663-680

${ }^{6}$ Kyle RF, Ellis TJ, Templeman DC. Surgical treatment of intertrochanteric hip fractures with associated femoral neck fractures using a sliding hip. J Orthop Trauma 2005; 19: 1-4

Seinsheimer F. Subtrochanteric Fractures of 300-306

${ }^{8}$ Waddell JP. Subtrochanteric Fractures of the Femur (1979). A review of 130 patients. J Trauma 1979; 19: 582-591

Prof. MUDr. Jan Bartoníček, DrSc. Vorstand der Klinik

Ortopedicko-traumatologická

klinika 3. LF UK

Šrobárova 50

10034 Praha 10

Česká republika

E-Mail: bartonic@fnkv.cz the Femur. J Bone Joint Surg [Am] 1978; 60: 